\title{
Das deutsche Kopulaverb sein und seine thailändischen Entsprechungen
}

\author{
Korakoch Attaviriyanupap (Nakhon Pathoom)
}

\begin{abstract}
Although German and Thai are typologically different from each other, both languages do have copulative constructions. The verb sein is the most important copular verb in German. Thai does have literary equivalents for this German verb but they involve different verbs. However, only pen and khu: are usually considered as Thai copular verbs. This study aims to compare the German verb sein in copulative constructions with pen and khw: The contrastive analysis is based on a bidirectional parallel corpus consisting of 12 Thai and 13 German contemporary short stories and their translation into the other language. Three questions are to be answered: 1) Which forms are found in Thai as equivalents to the German copular verb sein? 2) Which linguistic elements in German occur as equivalents of the Thai copulative constructions with pen and khw:? 3) How can the use of copular verbs in German and in Thai be described? The results of this study show that the equivalents of the German copulative constructions with sein are not only pen and khw: but also many other constructions. At the same time, the Thai copular verbs are often used differently and may be expressed in various German constructions and, especially in form of punctuations.
\end{abstract}

\section{$1 \quad$ Einleitung}

Kopulaverben sind grammatische Elemente, die die Funktion haben, gemeinsam mit einem nominalen Prädikativ das Prädikat zu bilden (cf. Bybee 2000: 806). Mit Kopulaverben bildet man im Allgemeinen statische Prädikate, jedoch sind sie nicht immer völlig bedeutungsleer, weshalb einige Sprachen über mehr als eine Kopula verfügen. In einigen Sprachen wird das Kopulaverb im Präsens weggelassen, kommt jedoch in anderen Tempora vor (z. B. Russisch). Im Deutschen werden üblicherweise sein, werden und bleiben als Kopulaverben bezeichnet. Das Verb sein gilt als das wichtigste Kopulaverb. Anders als in den meisten indoeuropäischen Sprachen, die über eine bestimmte Entsprechung des Verbs sein verfügen, gibt es im Thailändischen mehrere Entsprechungen von sein. Allerdings werden i.d. R. nur pen und khw: als Kopulaverben bezeichnet.

Nicht alle Konstruktionen mit sein im Deutschen werden als Kopula-PrädikativKonstruktionen bezeichnet. Es liegt keine einheitliche Klassifizierung vor. Aus der Perspektive des Thailändischen werden einige sein-Konstruktionen ausgeschlossen. In Kapitel 2.1 ist auf die Kriterien dafür einzugehen. 
Im Rahmen des vorliegenden Beitrags wird anhand des korpusbasierten Vergleichs einerseits alle Kopulakonstruktionen mit sein und ihre thailändischen Entsprechungen und andererseits die thailändischen Kopulakonstruktionen mit pen und khw: und ihre deutschen Entsprechungen analysiert. Folgende Fragen sind zu beantworten:

1. Welche thailändischen Entsprechungen der deutschen Kopula sein kommen im Korpus vor?

2. Welche sprachlichen Elemente treten im Deutschen als Entsprechungen der thailändischen Kopulakonstruktionen mit den Verben pen und khw: auf?

3. Wie lässt sich der Gebrauch der Kopulaverben im Deutschen und im Thailändischen beschreiben?

Zwar besteht das zu analysierende Korpus z. T. aus Übersetzungstexten, aber die vorliegende Studie ist nicht im Bereich der Übersetzungswissenschaft anzusiedeln. Die obige Fragestellung soll hingegen einen Beitrag zur Kontrastiven Lingustik leisten, weil deren Hauptziel es ist sprachübergreifende Konstruktionen zu analysieren.

\section{Kopulakonstruktionen mit sein im Deutschen und Kopulaverben im Thailändi- schen}

\subsection{Sein-Konstruktionen im Deutschen und deren Status als Kopulakonstruktion}

Obgleich das Vorkommen mit einem Prädikativ ein klares Indiz für ein Kopulaverb ist, ist die Klassifizierung der Form und Funktionen von Kopula-Konstruktionen mit sein im Deutschen in der gängigen Literatur häufig umstritten. Während Eisenberg (2013: 79) offen lässt, ob man das Verb sein bei einer lokalen Prädikation wie Karl ist hier, als eine Kopula betrachten oder einer anderen Verbkategorie zuordnen sollte, schreiben Heine/Kuteva (2002: 97-98, zit. in Hentschel/Vogel 2009: 455) einen solchen Fall der sog. „lokativen Kopula“ zu.

Helbig/Buscha (2001: 451) verweisen für Prädikative bei Kopulaverben auf folgende Formen: 1) Substantiv (oder substantivisches Pronomen) im Nominativ, 2) Adjektiv (oder Partizip I/II), 3) Präposition + Substantiv und 4) (Präposition +) Adverb. Das Prädikativ beim Verb sein kann in ein Attribut transformiert werden. Die als temporale oder lokale Adverbien auftretenden Glieder (Die Veranstaltung ist am Abend/abends; Der Lehrer ist in der Schule/dort) würden allerdings nicht als Prädikative, sondern als Adverbialbestimmung aufgefasst, was auf die Bedeutungsvarianten des Verbs sein zurückgeht (hier stattfinden bzw. sich befinden). Solche Konstruktionen seien deshalb aus der Gruppe der Kopula-Konstruktion auszuschließen.

Nach Helbig (2008: 81) werden nur zwei Konstruktionen einheitlich als Kopulakonstruktionen klassifiziert, nämlich die Konstruktion mit einer Nominalphrase im Nominativ wie $\mathrm{Pe}$ ter/ist/wird/bleibt Lehrer und diejenige mit einer Adjektivphrase wie Peter ist/wird/bleibt/gesund. Die Konstruktionen mit einer Lokalangabe wie Peter ist dort/in der Stadt und mit einer Genitivergänzung wie Peter ist guten Mutes werden m. E. hingegen nicht einheitlich behandelt. Ebenso unklar ist die Interpretation von Konstruktionen mit einer Temporalangabe wie Das Finale ist morgen/nächsten Samstag oder stofflicher Charakteristik wie Der Tisch ist aus Eichenholz. Helbig/Buscha (2001: 79) zufolge sehen einige Präpositionalphrasen, die gemeinsam mit sein verwendet werden, formal zwar wie eine lokale Situierung aus, weisen aber unterschiedliche Bedeutungen bzw. Funktionen zu, z. B. als Funktionsverb 
in Wendungen wie in Anwendung sein, in Betrieb sein, in Bewegung sein. In der IDSGrammatik Band I wird sein als „semantisch weitgehend ausgebleicht“ charakterisiert (Zifonun et al. 1997: 702). Häufig spricht man jedoch von vier verschiedenen Funktionen der Kopulasätze: Prädikation; Identität; Identifizierung und Spezifikation (cf. Higgins 1979, zit. in Geist 2006: 4). Die Differenzierungen lassen sich nicht an der Kopula an sich feststellen, sondern resultieren aus den Eigenschaften ihres Komplements.

In einer eigens durchgeführten Untersuchung wurde der Status von sein als Kopula aus der Perspektive des Thailändischen besprochen (cf. Attaviriyanupap 2017). Die Studie führte zum Ergebnis, die sein-Konstruktionen im Hinblick auf ihren Kopulastatus in drei Kategorien einzustufen. Diese reichen von ,eindeutig Kopula“ über „Kopula mit Einschränkung“ zum Status „keine Kopula“.

Diejenigen Konstruktionen, die als „eindeutig Kopula“ bezeichnet werden sollen, betreffen die Konstruktionen sein + NS oder NP (die Voraussetzung dafür jedoch war, dass zwischen Menschen eine Liebe bestand; Mein Mann und ich sind Schauspieler). Die Konstruktion sein + AdjP (Ihr Gesicht ist kreidebleich) bildet den Übergang zwischen dieser Kategorie und der nächsten, nämlich „Kopula mit Einschränkung“. Zu dieser Gruppe gehören noch die Konstruktionen sein + AdvP (Er ist nicht hier), PP (die Fenster waren zu) oder Partizipien (aber das ist irreführend; deren Taschen oft prall gefüllt sind). Es handelt sich bei der Konstruktion sein + Partizip II jedoch um die Passivkonstruktion, während die Perfektbildung mit sein (z. B. dass ich mir ständig als ihr Publikum vorgekommen bin) ausgeschlossen bleibt und nicht als Kopulakonstruktion zu bezeichnen ist. Auch andere restliche Konstruktionen mit sein + VP jeglicher Art lassen sich laut dieser Studie als „keine Kopula“ klassizifizieren. In der vorliegenden Untersuchung werden deshalb nur die Konstruktionen der ersten zwei Gruppen mitberücksichtigt.

\subsection{Kopulaverben im Thailändischen}

In gängigen Wörterbüchern wird sein stets mit drei Wörtern, pen, jù:, khw:, übersetzt. Bei Smyth (2002: 56-58), der vom englischen Verb to be ausgeht, liegt mi: noch als die vierte Form vor, nämlich als Entsprechung der englischen Konstruktionen there is und there are. Es ist jedoch anzumerken, dass pen und khu: überwiegend mit einem Substantiv oder einer Nominalphrase (NP) auftreten (cf. Pustet 2003: 47) und sich für das Thailändische eindeutig als Kopula bezeichnen lassen, während mit jù: und mi: eher eine lokale Prädikation gebildet wird. Iwasaki/Ingkaphirom (2005: 221-223) erwähnen im Hinblick auf Kopulaverben im Thailändischen nur pen und khw: Dabei wird pen nicht als Verb, sondern nur als sog. „SemiVerb“ behandelt, das darstellt, dass sich ein Gegenstand, eine Person oder ein Konzept in irgendeinem Zustand befindet. Ihnen zufolge fungiert khw: als Anknüpfer, der den Namen, das Etikett oder eine Definition eines Gegenstands, einer Person oder eines Konzeptes einführt. Es gibt darüber hinaus einen fließenden Übergang zwischen der im Deutschen als prototypisches Adjektiv bezeichneten Wortart und dem Verb. Adjektive wie z. B. di: (,gut'), lék (,klein`) können im Thailändischen sowohl attributiv als auch prädikativ gebraucht werden. Da bei prädikativem Gebrauch üblicherweise kein Kopulaverb auftritt, werden solche Konstruktionen als Zustandsverben bezeichnet, während sie üblicherweise als nicht-verbale Prädikate zugeordnet werden (cf. v. a. Henvegeld 1992). In dieser Hinsicht lässt sich ebenfalls 
eine Besonderheit der Kopula pen im Thailändischen feststellen, weil es ebenfalls weglassbar ist (z. B. nî̀: bâ:n chân DET + ,Haus' + ,ich ${ }^{\star}=$,Das ist mein Haus'). Diese Kopula kommt jedoch regelmäßig bei der Konstruktion mit einem nominalen Prädikativ und gelegentlich mit einer entsprechenden Form eines Adjektivs im Deutschen vor. Die Kopula pen ist nur notwendig, wenn es sich um physische oder mentale Bedingungen sowie einen semipermanenten Zustand einer Person oder eines Gegentandes handelt, z. B. pen bâj (,stumm'), pen sò:t (,ledig'), pen khâj (,fiebrig') oder pen sànǐm (,rostig') (cf. Iwasaki/Ingkaphirom 2005: 222).

Traditionell werden die beiden Kopulaverben im Thailändischen, nämlich pen und khu:, im Hinblick auf ihre Funktion unterschieden. Während khw: zur Identifizierung des Subjekts dient, weisen Konstruktionen mit pen die Charakterisierung des Satzsubjekts auf. Takahashi/Shinzato (2003) untersuchten die Unterschiede zwischen den Kopulasätzen mit khw: und denjenigen mit pen und ziehen den Schluss, dass sich die beiden thailändischen Kopulaverben durch ihre Art der Informationsverarbeitung unterscheiden. Die Kopula khu: ist mit einer schnellen, pen mit einem langsamen Informationsverarbeitungsprozess verbunden. Beim ersten Fall handelt es sich also um etwas Sensationelles, während im zweiten Fall die Informationsverarbeitung einen Denksprozess auslöst. Die Kopula pen impliziert deshalb längere Zeit für die Informationsverarbeitung (cf. ibd.: 144). Im alltäglichen Sprachgebrauch des Thailändischen kommt khu: im Vergleich zu pen nicht so häufig vor. Dies liegt anscheinend zum großen Teil auch daran, dass pen, ähnlich wie seine deutsche Entsprechung sein, multifunktional und im Gebrauch hochfrequent ist.

Jaratjarungkiat (2012) untersuchte die diachronische Entwicklung des Verbs pen im Thailändischen. Dabei wurde pen im Hinblick auf Wortarten, Funktionen, Bedeutungen sowie Gebrauchskontexte analysiert. Es wurde festgestellt, dass sich das Wort pen in 2 Typen kategorisieren lässt: lexikalisches Element und nicht-lexikalisches Element. Als lexikalisches Wort hat pen sechs Bedeutungen: ,leben', ,entstehen/geschehen', ,existieren`, ,können“, ,das Gefühl kennen‘ und ,in einer richtigen Phase sein'. Als nicht-lexikalisches Wort lässt sich pen zum einen als Kopula und zum anderen als Verbersatz bezeichnen. Das thailändische Verb pen weist ebenfalls sprachübergreifende Merkmale auf, weil Kopulaverben in verschiedenen Sprachen eine ähnliche Entstehungsgeschichte haben: Sie entstehen oft durch die Grammatikalisierung von Positions- und Bewegungsverben (cf. Geist/Rothstein 2007).

\section{$3 \quad$ Korpus und Analysenmethode}

Für die vorliegende Analyse wird das von Johansson (2003: 39) beschriebene Modell eines bidirektionalen parallelen Korpus verwendet, das sich aus Original- und Übersetzungstexten der zu vergleichenden Sprachen zusammensetzt. Das eigens zusammengestellte Korpus (ca. 234'500 Wörter) besteht aus 13 deutschen und 12 thailändischen zeitgenössischen Kurzgeschichten und deren Übersetzung in die jeweilige andere Sprache. Die deutschen und die thailändischen Texte sind inhaltlich und stilistisch vergleichbar. Es handelt sich bei den als Korpus zusammengestellten Texten um dasselbe literarische Genre (Kurzgeschichte). Die Texte spiegeln im Hinblick auf ihrer Entstehungszeit und Übersetzer den Sprachgebrauch der zeitgenössischen Literatur wider. Im Korpus sind vier verschiedene Texte vorhanden: deutsche Originaltexte, deutsche Übersetzungstexte, thailändische Originaltexte und thailändische 
Übersetzungstexte. Insgesamt umfasst das Korpus also 50 Texte, die zwischen 1980 und 2008 erschienen sind. In Tab.1 werden die wichtigsten Angaben über die Texte im Korpus dargestellt:

\begin{tabular}{|c|c|c|c|c|c|}
\hline Titel & Autor/in & Übersetzer/in(nen) & Jahr (O) & 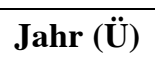 & Abk. ${ }^{1}$ \\
\hline Neues vom Norbert & Helmut Krauser & Pussadi Sikiau & 1990 & 2008 & HK \\
\hline Am See & Felicitas Hoppe & Ampha Otrakul & 1996 & 2008 & $\mathrm{FH}$ \\
\hline Töten & Daniel Kehlmann & $\begin{array}{l}\text { Aranya Rosenberg } \\
\text { Promnok }\end{array}$ & 2000 & 2008 & DK \\
\hline Zugfahrt & Julia Franck & Warangkana Siriwanont & 2000 & 2008 & $\mathrm{JF}$ \\
\hline $\begin{array}{l}\text { Erleuchtung durch } \\
\text { Fußball }\end{array}$ & Ralf Rothmann & Naruemon Ngaosuwan & 2001 & 2008 & RR \\
\hline Gestalten & Marcel Beyer & Atjana Saurer & 2001 & 2008 & $\mathrm{MB}$ \\
\hline $\begin{array}{l}\text { Die Freundin meines } \\
\text { Freundes }\end{array}$ & Nadja Einzmann & Suttichon Bhotirungsi & 2001 & 2008 & $\mathrm{NE}$ \\
\hline Unter Zeiten & Kevin Vennemann & Busarin Itsarachai & 2002 & 2008 & $\mathrm{KV}$ \\
\hline Kaltblau & Judith Hermann & $\begin{array}{l}\text { Choedchawee Sang- } \\
\text { chandr }\end{array}$ & 2003 & 2008 & $\mathrm{JH}$ \\
\hline Sibirien & Jenny Erpenbeck & Pornsan Wattanangkul & 2001 & 2008 & JE \\
\hline $\begin{array}{l}\text { Die Umgebung von } \\
\text { Blitzen }\end{array}$ & Silke Scheuermann & Janejira Sereeyothin & 2005 & 2008 & SS \\
\hline Sony Center & Ulrich Pelzer & $\begin{array}{l}\text { Korakoch Attaviriyanu- } \\
\text { pap }\end{array}$ & 2005 & 2008 & UP \\
\hline Abschied von Berlin & Arno Geiger & Anchalee Topeongpong & 2007 & 2008 & $\mathrm{AG}$ \\
\hline Die Highway-Familie & Sila Khomchai & $\begin{array}{l}\text { Kirsten Ritscher und } \\
\text { Heike Werner }\end{array}$ & 1993 & 2006 & SK \\
\hline $\begin{array}{l}\text { Begegnung auf der } \\
\text { Brücke }\end{array}$ & Phaithun Thanya & $\begin{array}{l}\text { Kirsten Ritscher und } \\
\text { Heike Werner }\end{array}$ & 1987 & 2006 & PT \\
\hline $\begin{array}{l}\text { Der Blumentopf am } \\
\text { Fenster }\end{array}$ & Win Liaowarin & $\begin{array}{l}\text { Kirsten Ritscher und } \\
\text { Heike Werner }\end{array}$ & 1996 & 2006 & WL \\
\hline Salmans kleine Welt & $\begin{array}{l}\text { Kanokphong } \\
\text { Songsomphan }\end{array}$ & $\begin{array}{l}\text { Kirsten Ritscher und } \\
\text { Heike Werner }\end{array}$ & 1991 & 2006 & KS \\
\hline $\begin{array}{l}\text { Der Topf, der nicht } \\
\text { mehr zu retten war }\end{array}$ & Anchan & $\begin{array}{l}\text { Kirsten Ritscher und } \\
\text { Heike Werner }\end{array}$ & 1990 & 2006 & An \\
\hline Ein guter Bürger & Seksan Prasertkul & $\begin{array}{l}\text { Kirsten Ritscher und } \\
\text { Heike Werner }\end{array}$ & 1980 & 2006 & SP \\
\hline Matsi & Sri Dao Rueang & $\begin{array}{l}\text { Kirsten Ritscher und } \\
\text { Heike Werner }\end{array}$ & 1985 & 2006 & Sri \\
\hline Die taube Greisin & $\begin{array}{l}\text { Praphasson Sewi- } \\
\text { kul }\end{array}$ & $\begin{array}{l}\text { Kirsten Ritscher und } \\
\text { Heike Werner }\end{array}$ & 1984 & 2006 & PS \\
\hline Die Erneuerung & $\begin{array}{l}\text { Wanit Charung- } \\
\text { kitanant }\end{array}$ & $\begin{array}{l}\text { Kirsten Ritscher und } \\
\text { Heike Werner }\end{array}$ & 1984 & 2006 & $\mathrm{WC}$ \\
\hline Die Bettler & Anchan & $\begin{array}{l}\text { Kirsten Ritscher und } \\
\text { Heike Werner }\end{array}$ & 1990 & 2006 & An2 \\
\hline $\begin{array}{l}\text { Abenddämmerung } \\
\text { auf dem Fluss }\end{array}$ & Assiri Thammachot & $\begin{array}{l}\text { Kirsten Ritscher und } \\
\text { Heike Werner }\end{array}$ & 1981 & 2006 & $\mathrm{AT}$ \\
\hline $\begin{array}{l}\text { Wie ein Staubkorn } \\
\text { auf der Erde }\end{array}$ & $\begin{array}{l}\text { Prichaphon } \\
\text { Bunchuai }\end{array}$ & $\begin{array}{l}\text { Kirsten Ritscher und } \\
\text { Heike Werner }\end{array}$ & 1980 & 2006 & $\mathrm{~PB}$ \\
\hline
\end{tabular}

Tabelle 1: Liste der Kurzgeschichten im analysierten Korpus

${ }^{1}$ Es handelt sich um die Abkürzung der Autorennamen, die als Kennzeichen der einzelnen Belege verwendet wird. 
Die vorliegende korpusbasierte Analyse beruht auf zwei Quellen der Kopula-Tokens im Korpus. Die erste Datengruppe betrifft die Daten der deutschen Kopulakonstruktionen mit sein und deren thailändischen Entsprechungen. Zunächst wurde in den deutschen Texten nach allen Belegen von Kopulakonstruktionen mit sein gesucht und diese nach ihrem Konstruktionstyp klassifiziert, anschließend wurde für die kontrastive Analyse nach ihren Entsprechungen im thailändischen Korpus gesucht. Die zweite Datengruppe bezieht sich auf sämtliche Tokens der thailändischen Kopulaverben pen und khw: die in der ersten Analyserunde nicht als Entsprechungen der deutschen Kopula sein festgestellt wurden. Jeder Beleg im Gesamtkorpus wird mit einer Abkürzung der Autorennamen, Nummer des Belegs und entweder O (Original) oder Ü (Übersetzung) gekennzeichnet. Zusätzlich dazu werden alle Belege mit entweder dt. oder th. kodiert, je nachdem ob es sich dabei um die Daten für die erste oder die zweite Analyserunde handelt.

\section{$4 \quad$ Ergebnisse}

\section{1 Überblick über die untersuchten Kopulakonstruktionen im Korpus}

Im deutschen Korpus lassen sich insgesamt 1'716 Kopulakonstruktionen mit sein finden, die im Hinblick auf deren Anzahl in jeder einzelnen Kurzgeschichte unterschiedlich auftreten. Es handelt sich um 977 Tokens in deutschen Originaltexten und 739 in deutschen Übersetzungstexten.

Im thailändischen Korpus liegen insgesamt 1'000 Tokens von pen und khw: vor, die nicht als thailändische Entsprechungen der deutschen Sein-Konstruktionen festgestellt werden. Dadurch, dass khw: im Thailändischen seltener Verwendung findet, ist die Anzahl der Tokens mit diesem thailändischen Kopulaverb erwartungsgemäß gering. 917 Tokens der thailändischen Kopulakonsstruktionen sind Konstruktionen mit pen (480 in deutschen Übersetzungsund 437 in thailändischen Originaltexten), während die Anzahl der Konstruktionen mit khu: bei insgesamt 83 Tokens liegt (50 in der deutschen Übersetzung und 33 in thailändischen Originaltexten).

\subsection{Thailändische Entsprechungen der deutschen Kopulakonstruktionen mit sein}

Im Korpus lassen sich die thailändischen Entsprechungen der deutschen Kopulakonstruktionen mit sein in 10 Gruppen kategorisieren:

- $\phi$ bzw. eine Nullform, d.h. keine formale Entsprechung lässt sich bestimmen, z. B. mé:krathây we:la: thî: jâ: sòkapròk (,obwohl' + ,Zeit' + RELATIVPRONOMEN + ,Großmutter väterlicherseits' + , schmutzig $\left.{ }^{6}\right)$ als Entsprechung von selbst wenn sie schmutzig war [JE-dt121_O];

- pen (th. Kopula), z. B. wâ: phî:akkhăw pen phî:anbâ:n (,dass‘ + ,sie‘ + KOP + ,Nachbar') als Entsprechung von als seien sie alle meine Nachbarn [SK-dt47_Ü];

- khw: (th. Kopula), z. B. nân khw: hè:tphǒn thî: thammaj chăn chû:a (DET + KOP + ,Grund' + RELATIVPRONOMEN + ,warum ‘ + ,ich + , glauben') als Entsprechung von Das ist der Grund, warum ich glaube, ... [WL-dt215_Ü]; 
- $\quad$ châj (AP: Antwortpartikel châj, einschließlich deren negierte Form mâj châj), z. B. khun kô: mâj châj khonbr:lin mǔ:ankan châj máj (,Sie' + PARTIKEL + NEG + ANTWORTPARTIKEL + ,Berliner ${ }^{6}+$, auch ${ }^{6}+$ ANTWORTPARTIKEL + FRAGEPARTIKEL) als Entsprechung von Sie sind auch kein Berliner, was? [AG-dt49_O]. Das Wort châj fungiert als Antwortpartikel im Thailändischen und entspricht der Antwortpartikel ja im Deutschen. Es gilt als Äquivalenz für die Äußerung: es ist so bzw. es stimmt so und weist deshalb Ähnlichkeit mit dem Kopulaverb pen auf. Im Thailändischen kann châj ebenfalls als pen chên nán (KOP-pen,+ wie $^{6}+$, so $\left.^{6}\right)$ paraphrasiert werden. Um eine Kopulakonstruktion mit NPs zu negieren, werden ebenfalls häufig Ausdrücke mit mâj châj verwendet;

- mi: (,haben“, ,besitzen“, ,existieren'), z. B. troך lû:kbìtpràtu: mi: ro:j khri:m (,an“ + ,Türklinke ${ }^{+}+$,existieren' + ,Spur ${ }^{\star}+$, Creme') als Entsprechung von An der Klinke waren noch Spuren ihrer Nachtcreme [RR-dt53_O];

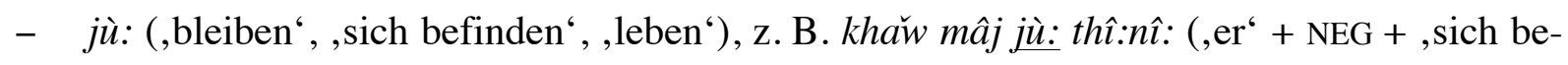
finden',+ ,hier $\left.{ }^{6}\right)$ als Entsprechung von er ist nicht hier [JH-dt272_O];

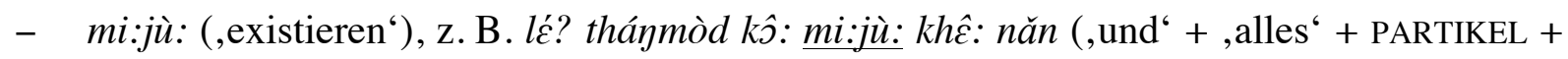
,existieren' + ,nur ${ }^{6}+$ DET) als Entsprechung von Und das ist alles [JH-dt285_O]. Es handelt sich bei dieser Entsprechung um die Kombination von mi: und jù:, die entweder genau in dieser Form oder als eine zweiteilige Verbform mi:...jù: auftritt. Während die Verben $m i$ : und ju: mehrere Bedeutungen haben können, beschränkt sich die Kombinationsform mi:jù: nur auf die Bedeutung ,existieren";

- KOP-ähnl. (die sich in vielen Sprachen ähnlich wie die Kopula verhaltenden Verben,

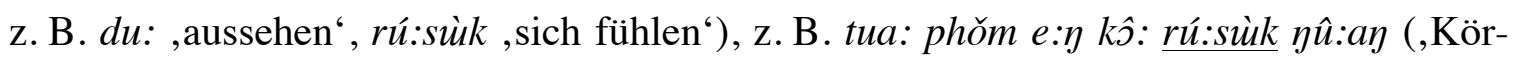

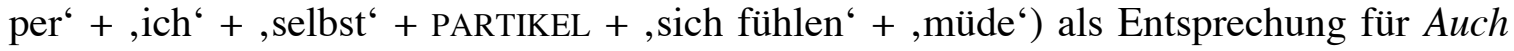
ich bin müde [KV-dt27_O]. Im Englischen werden die Entsprechungen solcher Verben nicht mit einem Adverb, sondern mit einem Adjektiv kombiniert (cf. z. B. she looks happy; she felt sad), weshalb sie morphosyntaktisch und semantisch Gemeinsamkeiten mit einer Kopula aufweisen;

- V div (diverse Verben), z. B. ich war auch einmal mit ... zusammen [AG-dt54_O] phǒm k̂s: khr:j nı:n kàp: .... (,ich' + PARTIKEL + TAM-experientiell +, schlafen' + ,mit');

- Marker div. (prä- und postverbale Marker der Temporalität, Aspektualität, Modalität und des Passivs), z. B. khonraw thù:k kàkhăy wáj (,man“ + PASSIVMARKER + , einsperren“ + TAM-perfektiv) als Entsprechung von man immer eingesperrt war [DK-dt61_O].

Die folgende Tabelle zeigt die Distribution aller thailändischen Entsprechungen der deutschen Konstruktionen mit sein:

\begin{tabular}{|l|r|r|r|r|r|r|r|r|r|r|}
\hline $\begin{array}{c}\text { Form der } \\
\text { Entsprechung }\end{array}$ & \multicolumn{1}{|c|}{$\varnothing$} & \multicolumn{1}{c|}{ pen } & khu: & châj & mi: & jù: & mi:jù: & $\begin{array}{c}\text { KOP- } \\
\text { ähnl.V }\end{array}$ & $\begin{array}{c}\text { V. } \\
\text { div. }\end{array}$ & $\begin{array}{c}\text { Marker } \\
\text { div. }\end{array}$ \\
\hline Anzahl & 834 & 421 & 71 & 47 & 90 & 71 & 19 & 49 & 53 & 61 \\
\hline Prozent & 48,60 & 24,53 & 4,14 & 2,74 & 5,24 & 4,14 & 1,11 & 2,86 & 3,09 & 3,55 \\
\hline
\end{tabular}

Tabelle 2: Entsprechungen von deutschen Kopulakonstruktionen mit sein im thailändischen Korpus

Ungefähr die Hälfte der thailändischen Entsprechungen des deutschen Verbs sein tritt im Korpus als Nullform ( $\varnothing$ ) auf. Der hohe Anteil ist auf zwei unterschiedliche Aspekte zurückzu- 
führen. Zum einen handelt es sich bei den untersuchten Texten nicht um wörtliche Übersetzungen. Vieles wird nur sinngemäß übertragen und müsste dadurch anders formuliert werden als die Strukturen in den Originaltexten. Deshalb kommt die Entsprechung des Verbs sein häufig als Nullform vor. Zum anderen ist es im Thailändischen möglich, auf die Verwendung des Kopulaverbs zu verzichten (s. Kapitel 2.2), weshalb es nicht erstaunlich ist, dass die Nullform auftritt, v. a. bei der Konstruktion mit Adjektiv. Auch verblose nominale Prädikate lassen sich unter dieser Form der thailändischen Entsprechung finden, z. B. phátsadù» hò: nán

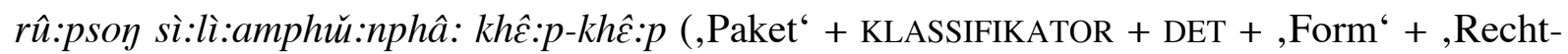
$\mathrm{eck}^{6}+$,schmal-schmal') als Entsprechung für Das Paket ist rechteckig und schmal [JHdt3_O].

Anhand der dargestellten Ergebnisse lässt sich feststellen, dass zwar ein großer Teil von der deutschen Kopula sein im Thailändischen als Nullform angegeben wird, dennoch entspricht sein im Deutschen mehrheitlich den thailändischen Kopulaverben oder zumindest vergleichbaren Verben bzw. Konstruktionen. Nur bei etwa 6,64 \% der thailändischen Entsprechungen (d. h. die beiden letzten Kategorien) handelt es sich um Entsprechungen, die nicht als „kopularelevant" zu bezeichnen sind.

\subsection{Deutsche Entsprechungen der thailändischen Kopulakonstruktionen mit pen und khu:}

Im Korpus lassen sich die deutschen Entsprechungen der thailändischen pen- und khu:Konstruktionen ebenfalls in 10 Formen kategorisieren:

- $\quad \phi$ bzw. eine Nullform, d. h. keine formale Entsprechung lässt sich bestimmen, weil in der Übersetung andere Konstruktionen verwendet werden oder etwas in der übersetzten Fassung weggelassen oder ergänzt wird, z. B. und schaut nach, als Entsprechung von loy

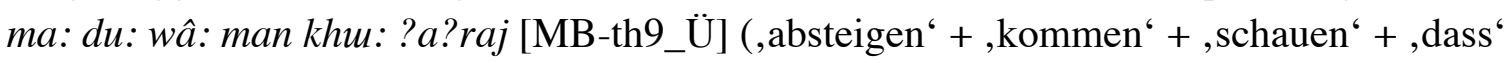
,$+ \mathrm{es}^{\text {' }}+\mathrm{KOP}+$, was' $^{\text {) }}$;

- NP, d. h. in der deutschen Fassung handelt es sich nicht um einen Satz mit Kopulaverb, sondern um eine Nominalphrase. Es können sowohl Substantive wie auch diverse Proformen sein, während im Thailändischen ein Satz mit einem der beiden Kopulaverben vorkommt, z. B. und dann Hügel als Entsprechung von lé? cà:k nàn cà? pen nr:n [DKth19_Ü] (,und' + ,dann` + TAM-prospektiv + KOP + ,Hügel' $)$;

- Verben Div. (Diverse Verben): Es handelt sich bei dieser Kategorie der deutschen Entsprechungen um verschiedene Verben, z. B. ... der Sala komplett aus Teakholz bestand als Entsprechung von săla: lăy ní: pen májsàk tháy lăy [WC-th21_O] (,Pavillon“ + , dies ${ }^{6}+\mathrm{KOP}+$, Teakholz $^{6}+$, ganz $\left.^{6}+\mathrm{KLF}\right)$. Interessanterweise handelt es sich zum einen darum, dass die wörtliche Übersetzung einiger deutscher Verben automatisch das thailändische Kopulaverb pen enthält, z. B. dt. gehören bedeutet auf Thai pen khว̌: $\eta$. Zum anderen sind Entsprechungen dieser Kategorie kopularelevante Verben, z. B. sich befinden, es gibt usw., die ähnlich wie lokative Kopulakonstruktionen fungieren. Diese beiden Verbtypen kommen mehrmals vor, während andere nur vereinzelt auftreten;

- Interpunktion: Unter den deutschen Entsprechungen der thailändischen Kopulakonstruktionen lässt sich nicht selten ein Satzzeichen finden, z. B. Sein ursprüngliches Ziel, New 


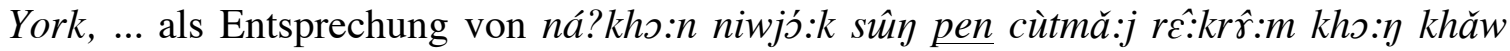
[AG-th1_Ü] (,Metropole' + ,New York ${ }^{`}+$ RELATIVPRONOMEN + KOP + ,Ziel ${ }^{`}+$,erst- ${ }^{\star}+$ ,beginnen' + , von $^{6}+$,er") oder ich hatte eine neue Entdeckung gemacht-meinen Körper als Entsprechung von lé? thî: chăn dâ:j ma: ?ì:k jày khw: waj să:w ?an ?ù:ap?àt [PB-

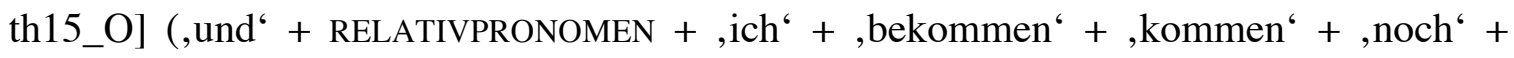
,Ding ${ }^{6}+\mathrm{KOP}+$, Alter $^{\star}+$, junge Frau $^{\star}+$ RELATIVPRONOMEN,+ mollig $\left.^{6}\right)$;

- AdjP (Adjektivphrasen): Im deutschen Text liegt keine Konstruktion mit dem Prädikat, sondern eine Adjektivphrase vor, z. B. wellig als Entsprechung von pen lû:k khlû:n (KOP + ,Kind' + ,Welle') [RR-th48_Ü]. Auch Partzipien, die attributiv verwendet werden, werden hier als Adjektive betrachtet. Ebenfalls bemerkenswert ist es, dass einige von diesen deutschen Adjektiven, die Kopula pen in ihrer wörtlichen thailändischen Übersetzung enthalten, weil es sich im Thailändischen um die Konstruktion KOP + NP handelt, z. B. dt. freundlich vs. th. pen mít (KOP + ,Freund`), dt. hölzern vs. pen má:j (KOP + ,Holz'). Dies ist sicherlich darauf zurückzuführen, dass der Status von Adjektiven als Wortart im Thailändischen an sich umstritten ist und die Konstruktion KOP + Adj im Thailändischen sich nur selten finden lässt;

- Adjunktor: In dieser Kategorie lassen sich zwei deutsche Adjunktoren, als und wie, die eine Nominalphrase einleiten, als Entsprechung der thailändischen Kopulakonstruktion feststellen, z. B. Als letztgeborene Tochter ... als Entsprechung von lì:n pen lû:ksă:w khon sùthá:j [An-th47_O] (,sie' + KOP + ,Tochter' + KLF + ,letzt-');

- PP (Präpositionalphrase): Auch Präpositionalphrasen ohne Verb treten als Entsprechung der thailändischen Kopulakonstruktion auf, z. B. Sie hat zum ersten Mal selber das Gefühl ... war der deutsche Originalsatz der thailändischen Übersetzung pen hǒn rê: $k$ thî: thr: mi: khwa:mrú:sùk wâ: [JH-th61_Ü] $\left(\mathrm{KOP}+, \mathrm{Mal}^{`}+\right.$, erst- ${ }^{`}+$ RELATIVPRONOMEN + ,sie + , haben' + ,Gefühl' + , dass'). Im Thailändischen findet man also einen komplexeren Satz mit einem Kopulaverb, während im Deutschen die Präpositionalphrase als Adverbial fungiert. Es sei anzumerken, dass die Präpositionalphrasen, die hier als deutsche Entsprechung bezeichnet werden, häufig eine ähnliche Struktur haben. Besonders häufig handelt es sich um die PP zum X.Mal, wie das oben genannte Beispiel zeigt;

- AdvP (Adverbphrase): Pararell zu der vorigen Kategorie lassen sich bei den Adverbphrasen, die als deutsche Entsprechung der thailändischen Kopulakonstruktion, besonders mit khu:, vorkommen, semantisch ähnliche sprachliche Elemente finden, nämlich solche Adverbien wie erstens, zuerst, zuletzt, z. B. Denn erstens gehört sich so etwas nicht als Entsprechung von hè:tphǒn khô: rê::k khu: nân mâj châj rû:ay thî: sǒmkhu:an [KVth2_Ü] (,Grund ‘ + KLF + , erst-` + KOP + , das ${ }^{\star}+$ NEG + ANTWORTPARTIKEL + , Geschich-

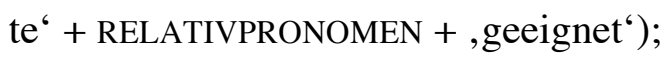

- Konjunktionen lassen sich als Entsprechung der Kopulakonstruktionen im Thailändischen finden. Es handelt sich vor allem um Nebensätze, die mit dass oder weil/da eingeleitet werden, z. B. und es wird darauf hinauslaufen, daß... als Entsprechung von phǒn

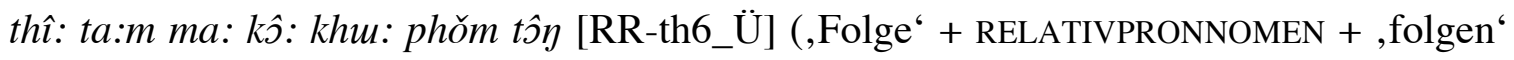

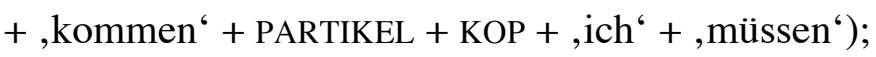


- Modalwörter treten ebenfalls als Entsprechung thailändischer Kopulakonstruktionen auf. Während diese im Deutschen als eine Konstituente im Satz erscheinen, weist das Thailändische eine Konstruktion eines komplexen Satzes mit der Kopula pen auf, z. B. viel-

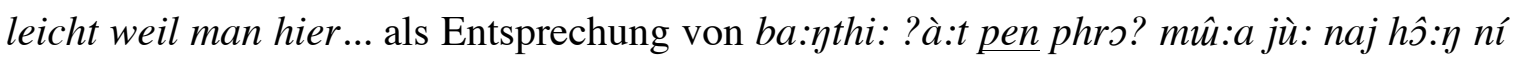

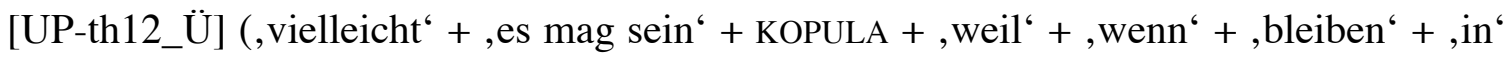
,+ dies $^{6}+$, Zimmer $\left.^{6}\right)$;

Die Verteilung der einzelnen Formen der deutschen Entsprechungen von pen und khw: wird in der folgenden Tabelle dargestellt:

\begin{tabular}{|l|l|l|l|l|}
\hline & \multicolumn{2}{l}{ pen } & khu: \\
\hline Deutsche Entsprechung & Anzahl & Prozent & Anzahl & Prozent \\
\hline$\varnothing$ & 146 & 15,92 & 17 & 20,48 \\
\hline NPs & 221 & 24,10 & 6 & 7,23 \\
\hline Verben div. & 183 & 19,96 & 14 & 16,87 \\
\hline Interpunktion & 72 & 7,85 & 23 & 27,71 \\
\hline AdjP & 89 & 9,16 & 0 & 0 \\
\hline Adjunktor & 84 & 9,16 & 2 & 2,41 \\
\hline PP & 72 & 7,85 & 2 & 2,41 \\
\hline AdvP & 21 & 2,29 & 12 & 14,46 \\
\hline Konjunktion & 13 & 1,42 & 7 & 8,43 \\
\hline Modalwörter & 16 & 1,74 & 0 & 0 \\
\hline
\end{tabular}

Tabelle 3: Entsprechungen von thailändischen Kopulakonstruktionen im deutschen Korpus

Die oben klassifizierten Formen treten im Korpus in unterschiedlichem Umfang auf. Während alle Formen bei Konstruktionen mit pen vorkommen, lässt sich kein Beleg von Adjektivphrasen oder Modalwörtern als Entsprechung der Kopulakonstruktion mit khu: finden. Bemerkenswert ist, dass die deutschen Entsprechungen ebenfalls ähnliche Strukuren aufweisen, und wie im Thailändischen das Kopulaverb weggelassen werden kann, wenn im Deutschen das Verb sein als Kopula nur die grammatische Funktion übernimmt und semantisch leer ist.

Vergleicht man die deutschen Entsprechungen der thailändischen Kopulaverben in Tab. 3 mit den thailändischen Entsprechungen der deutschen Kopulakonstruktionen mit sein in Tab. 2 lassen sich sowohl Gemeinsamkeiten als auch Unterschiede finden. Wenn man die Weglassbarkeit zunächst ausschließt, handelt sich in den meisten Fällen überwiegend um den Gebrauch anderer Verben, die unterschiedlichen Grad der Relevanz zu Kopulaverben aufweisen. Die wichtigste Form der Entsprechung von Kopulakonstruktionen, die m. E. nur in der deutschen Sprache auftritt, ist die Interpunktion.

\section{$5 \quad$ Schluss}

Obwohl sowohl das Deutsche als auch das Thailändische über Kopulaverben verfügen, verhalten sie sich vollkommen anders. Die Entsprechungen der deutschen Kopula sein müssen nicht die thailändischen Kopulaverben pen und khw: sein. Andere Konstruktionen liegen vor. Jedoch handelt es sich überwiegend um vergleichbare Konstruktionen, vor allem um kopulaähnliche Verben. Gleichzeitig werden die beiden thailändischen Kopulaverben häufig verwendet, während die entsprechenden Konstruktionen im Deutschen kein Prädikat bzw. keine Kopula enthalten. 
Die Ordinalia im Deutschen scheinen ebenfalls eine wichtige Form der Entsprechung thailändischer Kopulaverben zu sein, wie dies ersichtlich ist, wenn solche Phrasen wie zum ersten Mal, zum letzten Mal, erstens oder zuletzt häufig vorkommen, wo im Thailändischen pen und khu: verwendet werden. Dass Kopulaverben eher semantisch leer sind und zwei sich auf dasselbe beziehende Elemente miteinander verknüpfen, weist sicherlich Ähnlichkeit mit einer Reihung auf und entspricht der Funktion von Ordinalia.

Während es sich beim Nicht-Einsatz der Kopulaverben im Thailändischen eher um die Weglassbarkeit der Kopula oder die Verwendung kopulaähnlicher Verben handelt, stellen die deutschen Entsprechungen ein anderes Bild dar. Das Deutsche verfügt über mehr morphologische Mittel, weshalb unterschiedliche Konstruktionen als Entsprechungen der thailändischen Kopulaverben auftreten. Bemerkenswert ist dies vor allem bei der Verwendung der Komposita. Nicht selten kommt im Deutschen ein Kompositum als Entsprechung der thailändischen Struktur mit einem Substantiv vor, das von einem Relativsatz attribuiert wird, der wiederum eines der thailändischen Kopulaverben enthält. Hier kann man deshalb den Schluss ziehen, dass auch die Wortbildung im Deutschen die syntaktische Funktion einer Kopulakonstruktion übernimmt. Das Deutsche tendiert zur Nominalisierung, während dieses morphologische Mittel im Thailändischen kaum Verwendung findet.

Schließlich verfügt das Deutsche in der Schriftsprache über die Interpunktion, die ebenfalls die Funktion einer Kopula einnehmen kann. Die verschiedenen Satzzeichen bilden eine wichtige Gruppe der deutschen Entsprechungen thailändischer Kopulakonstruktionen mit pen und khw: Auch hier wird der Unterschied zwischen diesen beiden Sprachen verdeutlicht. Wenn man davon ausgeht, dass eine Kopula semantisch leer ist, können zwei Satzteile wie das Subjekt und das Prädikativum ebenfalls mit einem Satzzeichen miteinander verbunden werden. Vor allem das Komma, das häufig eine NP mit ihrem Attribut in Form einer Apposition einleitet, kann die Funktion eines Kopulaverbs sehr gut übernehmen. Im Thailändischen ist die Verwendung von Satzzeichen ungewöhnlich. Traditionell ist sie sogar verboten. Obgleich Satzzeichen heutzutage durch Einfluss von europäischen Sprachen in der thailändischen Sprache gelegentlich Verwendung finden, wird deren Einsatz noch als unschön oder sogar unakzeptabel empfunden.

Kopulaverben bilden m. E. zwar eine Verbkategorie, die sprachübergreifend definierbar ist, aber sie können sich ganz unterschiedlich verhalten. Deshalb wäre es für weitere Untersuchungen interessant, Kopulakonstruktionen sowie alle von deren möglichen Formen zur Umformulierung bzw. Umstrukturierung zu analysieren. Solche Erkenntnisse aus dem Bereich der Kontrastiven Linguistik könnten sowohl für die Übersetzung als auch für den Fremdsprachenunterricht nutzbar gemacht werden, egal ob es sich um Deutsch oder Thailändisch als Ausgangssprache handelt.

\section{Literatur}

Attaviriyanupap, Korakoch (2017): „Kopula oder keine Kopula? Das ist hier die Frage. Deutsche Kopulakonstruktionen mit sein aus der Perspektive des Thailändischen“. In: Akktramas, Pakini/Funk, Hermann/Traoré, Salifou (eds.): Deutsch als Fremdsprache im Spannungsfeld zwischen Globalisierung und Regionalisierung. Frankurt a. M./Bern/Wien, Lang: 351-369. 
Bybee, Joan (2000): „Verb“. In: Booij, Geert et al. (eds.): Morphologie. Ein Internationales Handbuch zur Flexion und Wortbildungen. Berlin/New York, de Gruyter: 794-808. (= HSK 17.1).

Eisenberg, Peter (2013): Grundriss der deutschen Grammatik Band 2: Der Satz. 4. Auflage. Stuttgart/Weimar: Metzler.

Geist, Ljudmila (2006): Die Kopula und ihre Komplemente. Zur Kompositionalität in Kopulasätzen. Tübingen: Niemeyer.

Geist, Ljudmila/Rothstein, Björn (eds.) (2007): Kopulaverben und Kopulasätze. Intersprachliche und intrasprachliche Aspekte. Tübingen: Niemeyer.

Heine, Bernd/Kuteva, Tania (2002): World Lexicon of Grammaticalization. Cambridge: Cambridge University Press.

Helbig, Gerhard/Buscha, Joachim (2001): Deutsche Grammatik: Ein Handbuch für den Ausländerunterricht. München: Langenscheidt.

Helbig, Gerhard (2008): „Zu den Kopulasätzen im Deutschen“. Deutsch als Fremdsprache 45: 81-90.

Hentschel, Elke/Vogel, Petra M. (2009): „Verb“. In: Hentschel, Elke/Vogel, Petra M. (eds.): Deutsche Morphologie. Berlin/New York, de Gruyter: 445-463.

Henvegeld, Kees (1992): Non-verbal Predication. Theory, Typology, Diachrony. Berlin/New York: de Gruyter.

Higgins, Roger (1979): The Pseudo-Cleft Construction in English. New York: Garland.

Iwasaki, Shoichi/Ingkaphirom, Preeya (2005): A Reference Grammar of Thai. Cambridge: Cambridge University Press.

Jaratjarungkiat, Sureenate (2012): The development of the word /pen/ in Thai. Unpublished $\mathrm{PhD}$ Dissertation, Chulalongkorn University.

Johansson, Stig (2003): "Contrastive lingusitics and corpora". In: Granger, Sylviane et al. (eds.): Contrastive Linguistics and Translation Studies. Amsterdam/New York, Rapodi: 31-44.

Pustet, Regina (2003): Copulas: Universals in the categorization of the lexicon. Oxford: Oxford University Press.

Smyth, David (2002): Thai. An Essential Grammar. London/New York: Routledge.

Takahashi, Kiyoko/Shinzato, Eumiko (2003): "On the Thai Copulas khuu1 and pen1: A Cognitive Approach". Proceedings of the $2^{\text {nd }}$ Seoul International Conference on Discourse and Cognitive Linguistics: Discourse and Cognitive Perspectives on Human Language: 131145.

Zifonun, Gisela et al. (1997): Grammatik der deutschen Sprache. Band I. Berlin/New York: de Gruyter. 